\title{
Cardiocirculatory Monitoring during Immediate Fetal-to-Neonatal Transition: A Systematic Qualitative Review of the Literature
}

\author{
Nariae Baik ${ }^{a, b} \quad$ Berndt Urlesberger ${ }^{a, b} \quad$ Bernhard Schwaberger ${ }^{a, b}$ \\ Thomas Freidll ${ }^{a} b$ Georg M. Schmölzer ${ }^{a, c, d}$ Gerhard Pichler ${ }^{a-d}$ \\ ${ }^{\mathrm{a}}$ Division of Neonatology and ${ }^{\mathrm{b}}$ Research Unit for Neonatal Micro- and Macrocirculation, Department of Pediatrics, \\ Medical University, Graz, Austria; ' Division of Neonatology, Department of Pediatrics, University of Alberta, and \\ ${ }^{d}$ Neonatal Research Unit, Royal Alexandra Hospital, Alberta Health Services, Edmonton, Alta., Canada
}

\section{Key Words}

Neonate $\cdot$ Transition $\cdot$ Heart $\cdot$ Circulation $\cdot$ Heart rate $\cdot$

Blood pressure $\cdot$ Cardiac function

\begin{abstract}
Background: The fetal-to-neonatal transition is a complex process that includes changes in cardiac and respiratory systems. Objective: The aim of this study is to review the different methods of cardiocirculatory monitoring during the immediate neonatal transition period. Methods: A systematic search of PubMed and Ovid Embase was performed using the following terms: infant, newborn, newborn infant, neonate, neonates, heart, cardiac, blood pressure, haemodynamic, hemodynamics, blood circulation, circulation, echocardiography, ultrasonography, sonography, electrocardiography, ECG, oximetry, pulse, pulse oximetry, monitoring, measurement, acclimatization, adaptation, transition, after birth and delivery room. Additional articles were identified by manual search of cited references. Only human studies describing cardiocirculatory monitoring during the first 15 min after birth were included. Results: Thirteen studies were
\end{abstract}

identified that described heart rate (HR). Additional five studies were identified that measured blood pressure. Four studies performed functional echocardiography during neonatal transition; two in addition to blood pressure monitoring and three in addition to HR monitoring. Conclusion: Routine HR monitoring using electrocardiography or pulse oximetry is used to evaluate adequate hemodynamic transition, and reference ranges have been established. Measuring blood pressure noninvasively though noncontinuously might be of some value in future, considering that the normative data have been established recently. Echocardiographic monitoring during the immediate transition period will improve the knowledge about cardiac function changes, but introduction in clinical routine remains questionable.

(c) 2014 S. Karger AG, Basel

\section{Introduction}

Immediate transition from intrauterine to extrauterine life (defined for this study as the first $15 \mathrm{~min}$ after birth) causes complex physiological processes affecting

\section{KARGER 125}

2014 S. Karger AG, Base

1661-7800/14/1072-0100\$39.50/0

E-Mail karger@karger.com

www.karger.com/neo
Gerhard Pichler, MD

Department of Pediatrics, Medical University of Graz

Auenbruggerplatz 30

AT-8036 Graz (Austria)

E-Mail pichler.gerhard@klinikum-graz.at 
Fig. 1. Flow diagram.

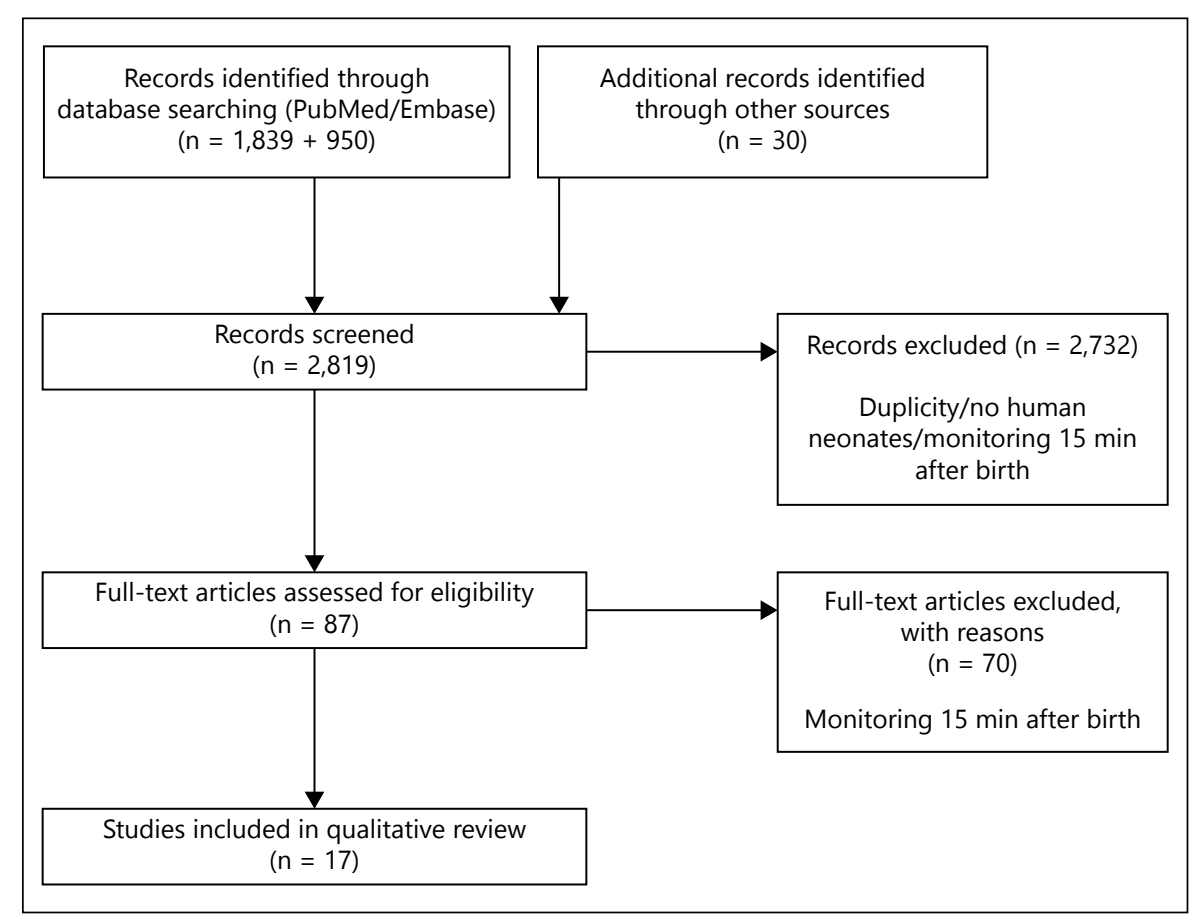

all vital organ systems [1]. During pregnancy the low-resistance placenta supplies the fetus with blood and oxygen. During fetal development, pulmonary vascular resistance remains high, the majority of the right ventricular output bypasses the lung and flows through the ductus arteriosus into the systemic circulation, whereas the left ventricular output (LVO) supplies the brain and the upper body. With the first breath immediately after birth, the pulmonary vascular resistance drops rapidly and the right ventricular output switches to the lungs [2]. In order to objectify the newborn's condition during this transition period, Virginia Apgar developed in 1953 a scoring system based on clinical assessments, which considers heart rate (HR), respiratory effort, reflex irritability, muscle tone and color [3]. However, a number of studies have already shown significant interobserver and intraobserver variability in clinical assessments of the newborn's condition $[4,5]$. Therefore, more objective and reliable methods to evaluate the newborn infants' condition in the delivery room is needed. According to the latest resuscitation guidelines of the European Resuscitation Council [6], the American Heart Association [7] and the International Liaison Committee on Resuscitation [8], it is recommended, besides the clinical evaluation, to monitor arterial oxygen saturation $\left(\mathrm{SpO}_{2}\right)$ by using pulse oximetry in the delivery room. This enables noninvasive continuous monitoring of the $\mathrm{SpO}_{2}$ as well as HR.

Cardiocirculatory Monitoring during

Neonatal Transition
Clinical assessment and monitoring with pulse oximetry unfortunately does not always give information about potentially compromised cardiocirculatory status influencing oxygen transport to tissue. Further cardiocirculatory monitoring might therefore be useful to obtain information to guide hemodynamic management for optimizing not only $\mathrm{SpO}_{2}$, but also cardiocirculation to improve oxygen transport to tissue.

The aim of the present systematic qualitative review was to identify studies describing noninvasive cardiocirculatory monitoring during the immediate transition time defined as the first 15 min after birth.

\section{Search Strategy and Study Selection Criteria}

Articles were identified using the stepwise approach specified in the Preferred Reporting Items for Systematic Reviews and Meta-Analyses Statement [9] (fig. 1).

\section{Search Strategy}

A systematic search was performed of PubMed and Embase to identify English-language articles published from July 1962 to July 2014. The studies had to address noninvasive cardiocirculatory monitoring in neonates during the first $15 \mathrm{~min}$ after birth. Search terms included: infant/newborn/newborn infant/neonate/neonates, 
Table 1. Cardiocirculatory monitoring immediately after birth

\begin{tabular}{llll}
\hline & HR & BP & Cardiac function \\
\hline Monitoring & $\begin{array}{l}\text { PO/ECG } \\
\text { continuous }\end{array}$ & $\begin{array}{l}\text { Doppler/oscillometric } \\
\text { noncontinuous }\end{array}$ & $\begin{array}{l}\text { echocardiography } \\
\text { noncontinuous }\end{array}$ \\
\hline Study design & observational & observational & observational \\
\hline Human studies & 13 & 5 & 4 \\
\hline
\end{tabular}

$\mathrm{PO}=$ Pulse oximetry

heart/cardiac/blood pressure/haemodynamic/hemodynamics/blood circulation/circulation, echocardiography/ultrasonography/sonography/electrocardiography/ ECG/oximetry/pulse/pulse oximetry, monitoring/measurement, and acclimatization/adaptation/transition/after birth/delivery room (Appendix 1,2). Additional published reports were identified through a manual search of references in retrieved articles and in review articles. Only human studies with cardiocirculatory monitoring within 15 min after birth were included. Studies starting cardiocirculatory monitoring $15 \mathrm{~min}$ after birth and animal studies were excluded.

\section{Study Selection}

Articles identified following literature review were evaluated independently by two authors (N.B., G.P.) for inclusion using the title and abstract. Where uncertainty remained regarding eligibility for inclusion, the full text was reviewed. Two reviewers independently identified relevant abstracts and critically appraised the full text of identified articles and assessed the methodological quality of included studies. All data were analyzed qualitatively. Data extraction included the characterization of study type, patient demographics, methods and results.

\section{Results}

The initial search detected 1,839 articles in PubMed and 950 in Ovid Embase; after removal of duplicates and rejection (e.g. no monitoring within the first $15 \mathrm{~min}$ after birth in human neonates; fig. 1), 17 studies were included in the qualitative review (table 1). All 17 studies performed cardiocirculatory monitoring during the first 15 min immediately after birth in human neonates. Thirteen studies placed importance on continuous measurement of HR (table 2). Three of them used only electrodes [10-
12], two used electrodes and pulse oximetry [13, 14], seven only pulse oximetry [15-21] and one did not mention the method of achieving HR [22]. Five studies described blood pressure (BP) during the defined transition period (table 3): one of them measured BP by the Doppler ultrasound method [23]; the other four used the oscillometric technique to achieve the results [11,24-26]. Four studies performed functional echocardiography during neonatal transition (table 4) $[11,12,20,25]$; two in addition to BP monitoring $[11,25]$ and three in addition to HR monitoring $[11,12,20]$.

\section{Discussion}

The following parameters of cardiocirculatory monitoring have been described during the immediate transition period: HR, BP and cardiac function.

\section{Heart Rate}

A rapid increase in HR immediately after birth is currently the most important parameter for adequate fetalto-neonatal transition [7]. If $\mathrm{HR}$ is $<100 / \mathrm{min}$, the international resuscitation guidelines suggest providing respiratory support [7]. However, HR $<100 / \mathrm{min}$ has been reported to be common in term and preterm infants [17, 19]. Animal and human observational studies have reported two different patterns of HR changes during the immediate transition period $[10,27]$. If cord clamping was performed before lung aeration occurred, a significant decrease in HR was observed [10]. However, if lung aeration was achieved before cord clamping, HR remained similar before and after cord clamping [10].

Changes in HR at birth are also affected by mode of delivery and maternal anesthesia. Several studies reported that vaginally delivered neonates had significantly higher HR compared to neonates born by caesarean section, which could also be an effect of maternal general anesthesia $[18,19]$. In addition, vaginally born neonates with maternal epidural anesthesia had initially higher HR compared to neonates whose mothers did not receive any analgesia [15]. Between vigorous and slightly depressed term vaginally delivered newborns, no difference in $\mathrm{HR}$ was observed [22].

In healthy term and late-preterm ( $>32$ weeks' gestation), several studies have reported that it can take several minutes until an increase in HR is observed [19]. In comparison, two different patterns of HR increase have been observed in infants requiring respiratory support $[28,29]$. Resuscitation, either with $100 \%$ oxygen or with 
Table 2. Heart rate monitoring in neonates immediately after birth

\begin{tabular}{|c|c|c|c|c|c|c|c|c|}
\hline Author/year & Gestation & $\begin{array}{l}\text { Mode of } \\
\text { delivery }\end{array}$ & $\begin{array}{l}\text { Method } \\
\text { used }\end{array}$ & Infants & $\begin{array}{l}\text { Tendency } \\
\text { first } 15 \mathrm{~min}\end{array}$ & $\begin{array}{l}\text { Respiratory } \\
\text { support }\end{array}$ & heart rate, bpm & $\begin{array}{l}\text { time after } \\
\text { birth, min }\end{array}$ \\
\hline Brady [10], 1962 & - & CS/VD & ECG & 112 & - & yes & $152^{\mathrm{a}} / 125^{\mathrm{b}}$ & 2 \\
\hline Bustos [22], 1975 & term & VD & - & 23 & increase & no & 170 & $3-5$ \\
\hline Meier-Stauss [13], 1990 & preterm/term & CS & $\mathrm{PO} / \mathrm{ECG}$ & 53 & - & yes & 158 & 2 \\
\hline Kopotic [16], 2002 & preterm & - & $\mathrm{PO}$ & 15 & - & yes & 110 & $2-3$ \\
\hline Kamlin [14], 2008 & preterm/term & - & $\mathrm{PO} / \mathrm{ECG}$ & 55 & - & yes & - & 2 \\
\hline Dawson [17], 2009 & preterm & - & $\mathrm{PO}$ & 126 & increase & yes & $<100$ & 1 \\
\hline Dawson [19], 2010 & preterm/term & $\mathrm{VD} / \mathrm{CS}$ & $\mathrm{PO}$ & 468 & increase & no & 96 & 1 \\
\hline van Vonderen [11], 2014 & term & $\mathrm{CS}$ & ECG & 24 & stable & no & 157 & 2 \\
\hline Noori [20], 2012 & term & VD & $\mathrm{PO}$ & 20 & decrease & no & 169 & $3-7$ \\
\hline
\end{tabular}

$\mathrm{CS}=$ Cesarean section; $\mathrm{VD}=$ vaginal delivery; $\mathrm{PO}=$ pulse oximetry. ${ }^{\mathrm{a}}$ Infants with onset of respiration before cord clamping. ${ }^{\mathrm{b}}$ Infants with onset of respiration after cord clamping.

Table 3. BP monitoring in neonates immediately after birth

\begin{tabular}{|c|c|c|c|c|c|c|c|}
\hline Author/year & Gestation & $\begin{array}{l}\text { Mode of } \\
\text { delivery }\end{array}$ & $\begin{array}{l}\text { Method } \\
\text { used }\end{array}$ & Infants & SBP & MBP & $\mathrm{DBP}$ \\
\hline Winberg [25], 1989 & term & CS & oscillometric & 16 & 67 & 45 & 32 \\
\hline Salihoglu [24], 2012 & preterm/term & $\mathrm{VD} / \mathrm{CS}$ & oscillometric & 982 & $63.82 \pm 12.24$ & $49.01 \pm 11.40$ & $37.76 \pm 11.63$ \\
\hline van Vonderen [11], 2014 & term & CS & oscillometric & 24 & $68.3 \pm 18.3$ & $51.2 \pm 15.4$ & $41.6 \pm 16.0$ \\
\hline
\end{tabular}

$\mathrm{SBP}=$ Systolic BP; MBP = mean BP; DBP = diastolic BP; CS = cesarean section; $\mathrm{VD}=$ vaginal delivery.

Table 4. Cardiac function monitoring in neonates immediately after birth

\begin{tabular}{|c|c|c|c|c|c|c|}
\hline \multirow[t]{2}{*}{ Author/year } & \multirow[t]{2}{*}{ Gestation } & \multirow{2}{*}{$\begin{array}{l}\text { Mode of } \\
\text { delivery }\end{array}$} & \multirow[t]{2}{*}{ Infants } & \multirow[t]{2}{*}{ Tendency first $15 \mathrm{~min}$} & \multicolumn{2}{|l|}{ First measurement } \\
\hline & & & & & $\mathrm{LVO}, \mathrm{ml} / \mathrm{kg} / \mathrm{min}$ & $\mathrm{LVSV}, \mathrm{ml} / \mathrm{kg}$ \\
\hline Noori [20], 2012 & term & VD & 20 & increase (LVO/LVSV) & $168 \pm 42$ & $1.01 \pm 0.23$ \\
\hline van Vonderen [11], 2014 & term & CS & 24 & increase (LVO/LVSV) & $151 \pm 47$ & $1.0 \pm 0.3$ \\
\hline van Vonderen [12], 2014 & term & $\mathrm{CS}$ & 24 & increase (LVO/LtoR shunting) & $161 \pm 50$ & - \\
\hline
\end{tabular}

$\mathrm{CS}=$ Cesarean section; $\mathrm{VD}=$ vaginal delivery; LtoR = left-to-right shunting via the ductus arteriosus.

Cardiocirculatory Monitoring during Neonatal Transition 
air in preterm neonates, had a similar effect on HR with a rising tendency [17]. Kopotic and Lindner [16] reported similar observations during resuscitation with $100 \%$ oxygen in a small number of extremely preterm neonates.

Several studies have compared HR monitoring using electrocardiography (ECG) with pulse oximetry and reported very good accuracy between ECG with pulse oximetry [14]. However, it has been reported that ECG placement can be applied more quickly compared to pulse oximetry [30].

An increase of $\mathrm{HR}$ during the first minutes after birth was observed in several studies in neonates without any need of medical support $[19,22]$ as well as in neonates with respiratory support $[17,18]$. Three studies reported different findings concerning the course of HR: Toth et al. [15] and Noori et al. [20] described a decreasing tendency of HR in vaginally delivered newborn infants without any medical support, and van Vonderen et al. $[11,12]$ reported in two separate studies no significant change of HR (measured by applying electrodes) in the first $10 \mathrm{~min}$ in term newborn infants. This has led to the development of reference ranges describing $\mathrm{HR}$ in healthy term and late-preterm ( $>32$ weeks' gestation) neonates without the need of any medical support in the first $10 \mathrm{~min}$ [19]. After midwifery-supervised uncomplicated vaginal birth, healthy neonates had significant lower values of $\mathrm{HR}$ and slower increase of $\mathrm{HR}$ in the first 3 min after birth compared to the international defined reference ranges [21].

\section{Blood Pressure}

BP is a composite of cardiac function and peripheral circulation as determined by cardiac output $(\mathrm{CO})$ and systemic vascular resistance, respectively, and is therefore an important indicator of appropriate circulation. Recently, the reference ranges of BP in preterm and term neonates during the immediate transition have been established [26]. Further studies have reported comparable values of BP changes during the immediate transition period in term infants without any need of medical support [11, 23-25]. BP values have been described to be lower in preterm infants compared to term infants and increase with increased gestational age $[24,26]$. Neonates after vaginal birth had significantly higher BP values compared to neonates born via caesarean section $[23,24,26]$. In addition, female neonates had higher mean systolic BP compared to male neonates [24]. Neonates recovering from asphyxia, exposed to maternal antihypertensive therapy and those born to mothers receiving thiopental within $4 \mathrm{~min}$ prior to delivery showed decreased BP, suggesting neonatal cardiocirculatory impairment immediately after birth [23].

Invasive BP measurement is not feasible during the immediate transition period and resuscitation. Therefore, the Doppler ultrasound and oscillometric method represent possibilities to measure $\mathrm{BP}$ noninvasively, whereby the oscillometric method is easier to apply than the Doppler ultrasound.

\section{Cardiac Function}

Stroke volume determines HR and cardiac output at beside. In term neonates, three studies reported an increase in LVO and left ventricular stroke volume (LVSV) within the first $10 \mathrm{~min}[11,12,20]$. Winberg et al. [25] reported higher LVO and LVSV at 15 min after birth in 16 term neonates delivered by caesarean section compared to vaginally born neonates. Two other recent studies reported that LVO and LVSV have similar patterns of increase in neonates born either vaginally or via caesarean section $[11,20]$. However, the reported difference in LVO and LVSV might be explained by the use of different techniques between the studies. In addition, shunting through a persistent ductus arteriosus has been described, becoming increasingly more left to right during the first $10 \mathrm{~min}$ after birth $[12,20]$.

\section{Limitations}

Evaluation of HR during immediate transition is recommended and already used to guide resuscitation. Pulse oximetry besides ECG is a noninvasive and continuous method to monitor the HR during immediate transition. It has been shown that ECG gets reliable signals more quickly than pulse oximetry [30]; however, applying ECG would be a further maneuver during neonatal resuscitation since the monitoring of $\mathrm{SpO}_{2}$ by applying pulse oximetry is already recommended in the latest resuscitation guidelines [6-8], and it provides continuous monitoring of both $\mathrm{SpO}_{2}$ and HR. Nevertheless, when using pulse oximetry, clinicians have to be aware of possible artefacts due to movements and manipulation.

Measuring BP noninvasively with the Doppler ultrasound as well as with the oscillometric method is absolutely feasible during neonatal transition, but the use of $\mathrm{BP}$ monitoring is limited due to the inability to measure BP continuously. Although these two methods measured comparable values of $\mathrm{BP}$, the oscillometric method is eas- 
ier to apply and therefore the practicability of the oscillometric method would be higher in the delivery room management setting.

Echocardiography has the ability to noninvasively measure cardiac functions during the immediate fetalto-neonatal transition. However, the use of echocardiography is limited due to (1) inability to measure cardiac function continuously, (2) dependence on ability and experience of the examiner, (3) parameters need to be defined prior measurements to limit time on echocardiography and not to interfere with resuscitation procedures, and (4) currently no reference values during immediate transition period are available.

\section{Conclusion}

Cardiocirculatory monitoring provides additional information and might be helpful to guide further hemodynamic management in the future. Ideally, cardiocirculatory monitoring should be noninvasive and provide reliable data continuously. Currently, noninvasive monitoring is routinely used to measure HR continuously during the immediate neonatal transition. New technologies of continuously measuring $\mathrm{BP}$ and cardiac function noninvasively might offer further insights in cardiovascular transition in the future.

In order to optimize the oxygen supply and to guide oxygen therapy, pulse oximetry is recommended in neonatal resuscitation guidelines. Although pulse oximetry and ECG enables monitoring HR continuously, it does not always provide information about potentially compromised cardiocirculatory status influencing oxygen transport to tissue.

Measuring BP noninvasively though noncontinuously might be of some value in the future, considering that reference rages have recently been established.

Echocardiographic monitoring during the immediate transition period will improve knowledge about cardiac function changes, but introduction in clinical routine remains questionable.

\section{Disclosure Statement}

The other authors have no conflicts of interest to disclose. The authors have no financial relationships relevant to this article to disclose. No external funding was secured for this study. G.M.S. is a recipient of a Banting Postdoctoral Fellowship, Canadian Institutes of Health Research and an Alberta Innovates - Health Solutions Clinical Fellowship.

Cardiocirculatory Monitoring during

Neonatal Transition

\section{Appendix 1}

Search strategy for PubMed: last performed July 8, 2014

No limits activated

\#1 MeSH descriptor newborn explode all trees (result: 619,194)

\#2 MeSH descriptor infant explode all trees (result: 977,013)

\#3 MeSH descriptor neonate explode all trees (result: 511,862)

\#4 ((\#1) OR \#2) OR \#3 (result: 1,098,441)

\#5 MeSH descriptor after birth explode all trees (result: 83,120)

\#6 MeSH descriptor transition explode all trees (result: 203,179)

\#7 MeSH descriptor delivery room explode all trees (result: 6,857)

\#8 ((\#5) OR \#6) OR \#7 (result: 291,327)

\#9 MeSH descriptor heart explode all trees (result: 1,144,844)

\#10 MeSH descriptor cardiac explode all trees (result: 1,339,030)

\#11 MeSH descriptor circulation explode all trees (result: 363,881)

\#12 MeSH descriptor hemodynamic explode all trees (result: 615,227)

\#13 MeSH descriptor blood pressure explode all trees (result: 502,516)

\#14 (((( \#9) OR \#10) OR \#11) OR \#12 OR) \#13 (result: $1,962,768$ )

\#15 MeSH descriptor monitoring explode all trees (result: 434,378)

\#16 MeSH descriptor measurement explode all trees (result: 454,193)

\#17 MeSH descriptor echocardiography explode all trees (result: 130,574)

\#18 MeSH descriptor electrocardiography explode all trees (result: 180,047)

\#19 MeSH descriptor ultrasonography explode all trees (result: 357,199)

\#20 MeSH descriptor pulse oximetry explode all trees (result: 14,814)

\#21 (((( \#15) OR \#16) OR \#17) OR \#18) OR \#19) OR \#20 (result: $1,352,440)$

\#22 ((( \#4) AND \#8) AND \#14) AND \#21 (result: 1,839) 


\section{Appendix 2}

Search strategy for Ovid Embase: last performed July 8, 2014

No limits activated

\#1 Newborn mp. [mp=title, abstract, subject headings, heading word, drug trade name, original title, device manufacturer, drug manufacturer, device trade name, keyword] (result: 359,885)

\#2 Infants mp. [mp=title, abstract, subject headings, heading word, drug trade name, original title, device manufacturer, drug manufacturer, device trade name, keyword] (result: 172,020)

\#3 Neonate mp. [mp=title, abstract, subject headings, heading word, drug trade name, original title, device manufacturer, drug manufacturer, device trade name, keyword] (result: 24,888)

\#4 (\#1 or \#2 or \#3) (result: 466,997$)$

\#5 After birth mp. [mp=title, abstract, subject headings, heading word, drug trade name, original title, device manufacturer, drug manufacturer, device trade name, keyword] (result: 29,102)

\#6 Transition mp. [mp=title, abstract, subject headings, heading word, drug trade name, original title, device manufacturer, drug manufacturer, device trade name, keyword] (result: 226,130)

\#7 Delivery room mp. [mp=title, abstract, subject headings, heading word, drug trade name, original title, device manufacturer, drug manufacturer, device trade name, keyword] (result: 2,454)

\#8 (\#5 or \#6 or \#7) (result: 256,926$)$

\#9 Heart mp. [mp=title, abstract, subject headings, heading word, drug trade name, original title, device manufacturer, drug manufacturer, device trade name, keyword] (result: 1,333,957)

\#10 Cardiac mp. [mp=title, abstract, subject headings, heading word, drug trade name, original title, device manufacturer, drug manufacturer, device trade name, keyword] (result: 498,642)

\#11 Circulation mp. [mp=title, abstract, subject headings, heading word, drug trade name, original title, device manufacturer, drug manufacturer, device trade name, keyword] (result: 156,431)

\#12 Blood pressure mp. [mp=title, abstract, subject headings, heading word, drug trade name, original title, device manufacturer, drug manufacturer, device trade name, keyword] (result: 331,179)

\#13 Hemodynamic mp. [mp=title, abstract, subject headings, heading word, drug trade name, original title, device manufacturer, drug manufacturer, device trade name, keyword] (result: 95,108)

\#14 (\#9 or \#10 or \#11 or \#12 or \#13) (result: $1,729,833$ )

\#15 Monitoring mp. [mp=title, abstract, subject headings, heading word, drug trade name, original title, device manufacturer, drug manufacturer, device trade name, keyword] (result: 539,028)

\#16 Measurement mp. [mp=title, abstract, subject headings, heading word, drug trade name, original title, device manufacturer, drug manufacturer, device trade name, keyword] (result: 511,899)

\#17 Echocardiography $\mathrm{mp}$. [mp=title, abstract, subject headings, heading word, drug trade name, original title, device manufacturer, drug manufacturer, device trade name, keyword] (result: 198,620)

\#18 Electrocardiography mp. [mp=title, abstract, subject headings, heading word, drug trade name, original title, device manufacturer, drug manufacturer, device trade name, keyword] (result: 71,786)

\#19 Pulse oximetry mp. [mp=title, abstract, subject headings, heading word, drug trade name, original title, device manufacturer, drug manufacturer, device trade name, keyword] (result: 10,415)

\#20 Ultrasonography $\mathrm{mp}$. [mp=title, abstract, subject headings, heading word, drug trade name, original title, device manufacturer, drug manufacturer, device trade name, keyword] (result: 94,036)

\#21 (\#15 or \#16 or \#17 or \#18 or \#19 or \#20) (result: $1,300,097)$

\#22 (\#4 and \#8 and \#14 and \#21) (result: 950) 


\section{References}

$>1$ Rudolph AM: Fetal and neonatal pulmonary circulation. Annu Rev Physiol 1979;41:383395.

$>2$ Noori S, Stavroudis TA, Seri I: Systemic and cerebral hemodynamics during the transitional period after premature birth. Clin Perinatol 2009;36:723-736, v.

-3 Apgar V: A proposal for a new method of evaluation of the newborn infant. Curr Res Anesth Analg 1953;32:260-267.

-4 O'Donnell CP, Kamlin CO, Davis PG, Carlin JB, Morley CJ: Clinical assessment of infant colour at delivery. Arch Dis Child Fetal Neonatal Ed 2007;92:F465-F467.

5 O’Donnell CP, Kamlin CO, Davis PG, Carlin JB, Morley CJ: Interobserver variability of the 5-minute Apgar score. J Pediatr 2006;149. 486-489.

6 Richmond S, Wyllie J: European Resuscitation Council Guidelines for Resuscitation 2010 Section 7. Resuscitation of babies at birth. Resuscitation 2010;81:1389-1399.

-7 Kattwinkel J, Perlman JM, Aziz K, Colby C, Fairchild K, Gallagher J, Hazinski MF, Halamek LP, Kumar P, Little G, McGowan JE, Nightengale B, Ramirez MM, Ringer S, Simon WM, Weiner GM, Wyckoff M, Zaichkin J: Part 15: neonatal resuscitation: 2010 American Heart Association guidelines for cardiopulmonary resuscitation and emergency cardiovascular care. Circulation 2010; 122:S909-S919.

$>8$ Wyllie J, Perlman JM, Kattwinkel J, Atkins DL, Chameides L, Goldsmith JP, Guinsburg R, Hazinski MF, Morley C, Richmond S, Simon WM, Singhal N, Szyld E, Tamura M, Velaphi S; Neonatal Resuscitation Chapter Collaborators: Part 11: neonatal resuscitation: 2010 International Consensus on Cardiopulmonary Resuscitation and Emergency Cardiovascular Care Science with Treatment Recommendations. Resuscitation 2010;81(suppl 1):e260-e287.

$\checkmark 9$ Moher D, Liberati A, Tetzlaff J, Altman DG; PRISMA Group: Preferred reporting items for systematic reviews and meta-analyses: the PRISMA statement. Int J Surg 2010;8:336-341.
$>10$ fetus and newborn infant during labor, delivery, and the immediate neonatal period. Am J Obstet Gynecol 1962;84:1-12.

11 van Vonderen JJ, Roest AA, Siew ML, Blom NA, van Lith JM, Walther FJ, Hooper SB, Te Pas AB: Noninvasive measurements of hemodynamic transition directly after birth. Pediatr Res 2014;75:448-452.

12 van Vonderen JJ, Te Pas AB, Kolster-Bijdevaate C, van Lith JM, Blom NA, Hooper $\mathrm{SB}$, Roest AA: Non-invasive measurements of ductus arteriosus flow directly after birth. Arch Dis Child Fetal Neonatal Ed 2014;99. F408-F412.

13 Meier-Stauss P, Bucher HU, Hurlimann R, Konig V, Huch R: Pulse oximetry used for documenting oxygen saturation and right-toleft shunting immediately after birth. Eur J Pediatr 1990;149:851-855.

14 Kamlin CO, Dawson JA, O’Donnell CP, Morley CJ, Donath SM, Sekhon J, Davis PG: Accuracy of pulse oximetry measurement of heart rate of newborn infants in the delivery room. J Pediatr 2008;152:756-760.

15 Toth B, Becker A, Seelbach-Gobel B: Oxygen saturation in healthy newborn infants immediately after birth measured by pulse oximetry. Arch Gynecol Obstet 2002;266:105-107.

16 Kopotic RJ, Lindner W: Assessing high-risk infants in the delivery room with pulse oximetry. Anesth Analg 2002;94:S31-S36.

17 Dawson JA, Kamlin CO, Wong C, Te Pas AB, O'Donnell CP, Donath SM, Davis PG, Morley $\mathrm{CJ}$ : Oxygen saturation and heart rate during delivery room resuscitation of infants $<30$ weeks' gestation with air or $100 \%$ oxygen. Arch Dis Child Fetal Neonatal Ed 2009;94: F87-F91

18 Gonzales GF, Salirrosas A: Pulse oxygen saturation and neurologic assessment in human neonates after vaginal and cesarean delivery. Int J Gynaecol Obstet 1998;63:63-66.

19 Dawson JA, Kamlin COF, Wong C, Te Pas AB, Vento M, Cole TJ, Donath SM, Hooper $\mathrm{SB}$, Davis PG, Morley CJ: Changes in heart rate in the first minutes after birth. Arch Dis Child Fetal Neonatal Ed 2010;95:F177-F181.

-20 Noori S, Wlodaver A, Gottipati V, McCoy M, Schultz D, Escobedo M: Transitional changes in cardiac and cerebral hemodynamics in term neonates at birth. J Pediatr 2012;160: 943-948.
21 Smit M, Dawson JA, Ganzeboom A, Hooper $\mathrm{SB}$, van Roosmalen J, Te Pas AB: Pulse oximetry in newborns with delayed cord clamping and immediate skin-to-skin contact. Arch Dis Child Fetal Neonatal Ed 2014;99:F309-F314.

22 Bustos R, Bejar R, Arroyave H, Jacomo AJ, Burghi M, Ramirez F, Cordano MC, Curbelo V, Guayasamin O, Minetti R, Guemberena L, Caldeyro-Barcia R: Heart rate in fetuses and neonates in normal conditions and with mild depression. J Perinat Med 1975;3:172-179.

23 Marx GF, Cabe CM, Kim YI, Eidelman AI: Neonatal blood pressures. Anaesthesist 1976; 25:318-322.

24 Salihoglu O, Can E, Beskardes A, Koc BS, LhanTan I, Can G, Hatipoglu S: Delivery room blood pressure percentiles of healthy, singleton, liveborn neonates. Pediatr Int 2012; 54:182-189.

25 Winberg P, Jansson M, Marions L, Lundell BP: Left ventricular output during postnatal circulatory adaptation in healthy infants born at full term. Arch Dis Child 1989;64:13741378.

26 Pichler G, Cheung PY, Binder C, O’Reilly M, Schwaberger B, Aziz K, Urlesberger B, Schmolzer GM: Blood pressure in term and preterm infants immediately after birth. Abstract E-PAS 2014:4655.4

27 Bhatt S, Alison BJ, Wallace EM, Crossley KJ, Gill AW, Kluckow M, Te Pas AB, Morley CJ, Polglase GR, Hooper SB: Delaying cord clamping until ventilation onset improves cardiovascular function at birth in preterm lambs. J Physiol 2013;591:2113-2126.

28 Yam CH, Dawson JA, Schmolzer GM, Morley CJ, Davis PG: Heart rate changes during resuscitation of newly born infants $<30$ weeks gestation: An observational study. Arch Dis Child Fetal Neonatal Ed 2011;96:F102-F107.

29 Palme-Kilander C, Tunell R: Pulmonary gas exchange during facemask ventilation immediately after birth. Arch Dis Child 1993;68: $11-16$.

30 Katheria A, Rich W, Finer N: Electrocardiogram provides a continuous heart rate faster than oximetry during neonatal resuscitation. Pediatrics 2012;130:e1177-e1181. 